\title{
Holistic health promoting food preparations from ayurveda
}

Vivek Kumar Dubey, Manas Ranjan Hota and Seema Pandey

Received : 10.04.2018; Accepted : 13.04.2018

See end of the Paper for authors' affiliation

Correspondence to :

Vivek Kumar Dubey Govt. Ayurveda College and Hospital, Bilaspur (C.G.) India Email : mangalpatil43@gmail. com
-Abstract : Ayurveda the science of life is serving the mankind since more than 5000 yeasrs. It has two main objectives, maintaining the health of healthy and alleviating the disease of diseased one. To maintain and promote the health food plays a central role. It is not just the ingredients but the the methods of preparation of food is crucial to provide optimum nutrition. Ayurvedic seers have though in depth and described in detail about how to make food most assimiable to the system. For this the food preperations have been categorized in twelve main groups and each group has its own advantages and specific health benefits. With the incresing prevelance of fast food culture and unhealthy food practices, the incidence of sevral life threatening diseases such as cancer, diabetes, hypertension are goes on increasing, not only that but our psychological status is also closely associated with our food habits. Unhealthy food habits are known cause for anxiethy, depression etc. and food modification is one of the important part of treatment in all such diseases. In this scenario, it becomes more important to know and search the solution from our ancient wisdom. The description from Ayurveda classics may be helpful for us to know the appropriate food practices which are suitable for our physical and psychological health. The presentation will discuss in detail about the various categories of food (Ahara varga) and its preparation methods along with the impact on our health

- Key words : Ayurveda, Ahara varga, Food

- How to cite this paper : Dubey, Vivek Kumar, Hota, Manas Ranjan and Pandey, Seema (2018). Holistic health promoting food preparations from ayurveda. Internat. J. Agric. Engg., 11(Sp. Issue) : 116-120, DOI: 10.15740/HAS/IJAE/11.Sp. Issue/116-120. 